\title{
Impact of Vonoprazan Triple-Drug Blister Packs on H.pylori Eradication Rates in Japan: Interrupted Time Series Analysis
}

\author{
Hisato Deguchi · Hajime Yamazaki (D) - Tsukasa Kamitani • \\ Yosuke Yamamoto $\cdot$ Shunichi Fukuhara
}

Received: March 18, 2021 / Accepted: May 7, 2021 / Published online: June 6, 2021

(C) The Author(s) 2021

\begin{abstract}
Introduction: Helicobacter pylori eradication therapy requires a complex prescribing schedule combining clarithromycin, amoxicillin, and a proton-pump inhibitor (PPI) or potassiumcompetitive acid blocker (P-CAB, vonoprazan). To reduce the burden of complex prescribing and increase adherence, a vonoprazan tripledrug blister pack comprising all three medications was launched in June 2016. This study
\end{abstract}

Supplementary Information The online version contains supplementary material available at https:// doi.org/10.1007/s12325-021-01784-w.

H. Deguchi · T. Kamitani · Y. Yamamoto Department of Healthcare Epidemiology, School of Public Health in the Graduate School of Medicine, Kyoto University, Kyoto, JapanT. Kamitani e-mail: kamitani.tsukasa.8w@kyoto-u.ac.jp Y. Yamamoto e-mail: yamamoto.yosuke.5n@kyotou.ac.jp

H. Deguchi Japan Medical Office, Takeda Pharmaceutical Company Limited, Tokyo, Japan e-mail: deguchi.hisato.33r@kyoto-u.jp

H. Yamazaki $(\bowtie) \cdot$ S. Fukuhara

Section of Clinical Epidemiology, Department of Community Medicine, Graduate School of Medicine, Kyoto University, 54 Kawahara-cho, Syogoin, Sakyo-ku, Kyoto 606-8507, Japan e-mail: yamazaki-myz@umin.ac.jpS. Fukuhara e-mail: fukuhara.shunichi.6m@kyoto-u.jp aimed to assess the impact of the combination blister pack on eradication success rate in Japan immediately after launch.

Methods: We performed an interrupted time series analysis using a large administrative claims database of 7,300,000 insured individuals. We identified 36,570 patients who received first-line clarithromycin triple therapy from June 2015 to May 2016 (prelaunch) and 35,721 who received the same therapy from July 2016 to June 2017 (post-launch). The primary outcome was the success rate of clarithromycin triple therapy and the secondary outcomes were proportion of vonoprazan use and proportion of combination blister pack use.

Results: The success rate of clarithromycin triple therapy increased by $2.44 \%$ (95\% confidence interval $[\mathrm{CI}] 1.36-3.52 ; P<0.0001)$ after the launch of the vonoprazan triple-drug blister pack. The proportion of vonoprazan use and proportion of combination blister pack use increased by $12.7 \% \quad(95 \%$ CI $10.0-15.3$; $P<0.0001)$ and $29.2 \%$ (95\% CI 25.4-32.9; $P<0.0001)$, respectively.

Conclusions: Launch of the vonoprazan tripledrug blister pack had a significant impact on the success rate of clarithromycin triple therapy, with greater proportions of vonoprazan and combination blister pack use. Introducing an easy-to-use formulation may be effective in changing prescribing practice and subsequent patient outcomes. 
Keywords: Drug packaging; Drug therapy; Helicobacter pylori; Interrupted time series analysis; Proton-pump inhibitors

\section{Key Summary Points}

Why carry out this study?

H. pylori eradication therapy requires a complex prescribing schedule combining clarithromycin, amoxicillin, and a protonpump inhibitor or a potassiumcompetitive acid blocker such as vonoprazan

To reduce the burden of complex prescribing and increase adherence, a vonoprazan triple-drug blister pack comprising all three medications was launched in June 2016

This study aimed to assess the impact of the combination blister pack on eradication success rate in Japan immediately after launch through analysis of a large administrative claims database

What was learned from the study?

The success rate of clarithromycin triple therapy increased by $2.44 \%$ after launch of the vonoprazan triple-drug blister pack. The proportion of vonoprazan use and proportion of combination blister pack use increased by $12.7 \%$ and $29.2 \%$, respectively

Launch of the vonoprazan triple-drug blister pack had a significant impact on the success rate of clarithromycin triple therapy, with greater proportions of vonoprazan and combination blister pack use. Introducing an easy-to-use formulation may be effective in changing prescribing practice and subsequent patient outcomes

\section{DIGITAL FEATURES}

This article is published with digital features, including a summary slide, to facilitate understanding of the article. To view digital features for this article go to https://doi.org/10.6084/ m9.figshare.14547465.

\section{INTRODUCTION}

Helicobacter pylori affects $50 \%$ of the world's population and is associated with peptic ulcer, mucosa-associated lymphoid tissue (MALT) lymphoma, and stomach cancer [1-6]. Clinical practice guidelines recommend $H$. pylori eradication for patients with peptic ulcers and MALT lymphoma [4-6]. Moreover, several randomized controlled trials (RCTs) and meta-analyses have shown that eradication therapy reduces the risk of developing gastric cancer [7-16].

Clarithromycin triple therapy, comprising a proton-pump inhibitor (PPI), clarithromycin, and amoxicillin or metronidazole, is the most common $H$. pylori eradication regimen worldwide [5]. However, the success rate of clarithromycin triple therapy has been declining with the rise in clarithromycin-resistant $H$. pylori [17-20]. Vonoprazan is a potassium-competitive acid blocker (P-CAB) launched in February 2015. It demonstrated noninferiority in a double-blind, randomized, phase 3 trial and subsequent superiority in an additional analysis versus lansoprazole; eradication rates were 92.6\% (95\% confidence interval [CI] 89.2-95.2) vs. $75.9 \%$ (95\% CI 70.9-80.5), respectively [21].

Clarithromycin triple therapy requires a complex prescribing schedule. To reduce the burden of complex prescribing and increase drug compliance, combination blister packs of clarithromycin triple therapy, which include a PPI (lansoprazole or rabeprazole), were introduced in Japan by 2014. Subsequently, a tripledrug blister pack including vonoprazan $\left(\right.$ VONOSAP $\left.^{\circledR}\right)$ was launched on June 7, 2016, so that complex eradication therapy could be easily and accurately administrated [22].

In vonoprazan triple-drug blister packs, daily doses are combined in one "sheet" and seven sheets are prescribed for 1 week (Fig. 1). 


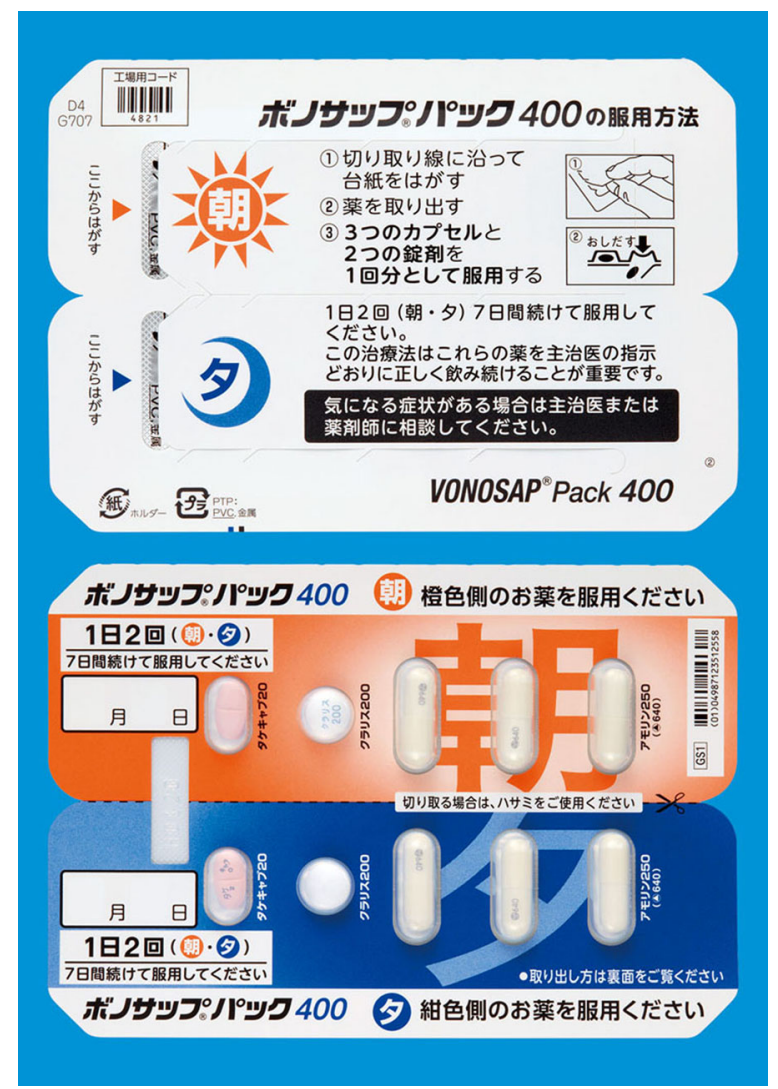

Fig. 1 Vonoprazan triple-drug blister pack (VONO$\mathrm{SAP}^{\circledR}$ ): Contains vonoprazan, clarithromycin, and amoxicillin (from left to right)

Additionally, medicines to be taken in the morning and evening are color-coded in the center. In a retrospective study looking at lansoprazole triple therapy, the eradication success rate was higher in the triple-drug blister pack group than in the separate-tablets group [23]. Conversely, the eradication success rate was the same in another retrospective study that examined vonoprazan triple therapy [24]. Moreover, there was no significant difference between the two groups in RCTs $[25,26]$. It is possible that the RCTs were affected by the Hawthorne effect because not only the blister pack group but also the separate-tablets group had good compliance; therefore, the full impact of the combination blister pack on the success rate of clarithromycin triple therapy remains unclear. It has been hypothesized that the launch of the vonoprazan triple-drug blister pack in June 2016 increased the proportion of vonoprazan and combination blister pack use, which may have improved $H$. pylori eradication success rates in Japan as a whole. However, no studies have evaluated whether launch of the vonoprazan triple-drug blister pack has increased the $H$. pylori eradication success rate. The present study aimed to assess the immediate change in eradication success rate of clarithromycin triple therapy before and after launch of the vonoprazan triple-drug blister pack.

\section{METHODS}

\section{Study Design}

As a primary outcome, an interrupted time series (ITS) analysis was conducted to examine changes in clarithromycin triple therapy success rate. For secondary endpoints, the proportion of vonoprazan use and proportion of triple-drug blister pack use were investigated. ITS analysis is considered to be within the highest tier of hierarchy in terms of quasi-experimental design and is useful in assessing the impact of interventions/reforms compared with a simple pre-post design [27]. One of the strengths of ITS analysis is that it is generally unaffected by typical confounding variables, which remain fairly constant, including clarithromycin-resistant $H$. pylori or baseline drug compliance, as these only change relatively slowly over time and are normally taken into account when modelling the underlying long-term trend [28]. ITS analysis is considered to be particularly effective for evaluation of population-level interventions, such as in cases where no control or comparison group is available [28]. We assessed whether the point at which the vonoprazan triple-drug blister pack was launched (June 2016) was associated with immediate increases (a change in the intercept) at the time of interruption or a change in the trajectory over time (the slope). Other triple-drug blister packs containing PPIs such as lansoprazole or rabeprazole were available by 2014 and vonoprazan itself was introduced in February 2015. Therefore, the intervention of this study is 
considered to be the launch of the vonoprazan triple-drug blister pack only.

\section{Patients}

We utilized data from a large health insurance claims database provided by JMDC Inc. (Tokyo, Japan). This database contains hospital outpatient, hospital inpatient, and pharmacy data from more than 90 employee-based health insurance societies; it includes data from approximately 7,300,000 insured Japanese individuals (representing around 6\% of the entire population of Japan). For each person, the JMDC database includes an enciphered personal identifier, family identifier, age, sex, diagnoses, medical services, and drugs prescribed. Enciphered personal identifiers were used to link claims data from different medical institutions and pharmacies. The investigators could only access anonymized information from the JMDC database; therefore, in accordance with the Japanese Ethical Guidelines, institutional ethics approval and informed consent were not required.

In this study, patients who had records for prescription of first-line clarithromycin triple therapy (clarithromycin, amoxicillin, and a PPI or vonoprazan) between June 1, 2015 and July 31, 2017 were included. Vonoprazan was launched before this period on February 26, 2015, and the vonoprazan triple-drug blister pack was launched on June 7, 2016. Patients who had records for prescriptions of second-line therapy (metronidazole, amoxicillin, and a PPI) before receiving first-line therapy were excluded.

\section{Success Rate of Clarithromycin Triple Therapy Against $\mathrm{H}$. pylori (Primary Endpoint)}

The only first-line $H$. pylori eradication therapy covered by insurance in Japan is a combination of clarithromycin, amoxicillin, and a PPI or vonoprazan. If patients fail first-line clarithromycin triple therapy then a combination of metronidazole, amoxicillin, and a PPI or vonoprazan is provided as second-line eradication therapy and this is the only regimen covered by insurance. Because H. pylori infection test results are not available in the database, a previously used definition was applied $[19,29]$. Specifically, failure of clarithromycin triple therapy was defined as the prescription of second-line therapy after completion of firstline clarithromycin triple therapy. Similarly, the repeat administration of first-line clarithromycin triple therapy was defined as failure of clarithromycin triple therapy. Successful clarithromycin triple therapy was defined as the prescription of first-line clarithromycin triple therapy that did not require the repeat of firstline therapy or the receipt of a second line of treatment. The monthly success rate was based on receipt of first-line therapy each month and success or failure was determined by receipt or not of second-line therapy after that.

\section{Proportion of Vonoprazan Use to Overall PPIs and P-CAB Use for $H$. pylori Eradication (Secondary Endpoint)}

The use of vonoprazan was defined as the receipt of a vonoprazan first-line triple-therapy regimen comprising clarithromycin, amoxicillin, and vonoprazan, or the receipt of vonoprazan triple-drug blister pack. Data used for the denominator were those with recorded prescriptions for any first-line clarithromycin triple therapy. This was assessed because the launch of the vonoprazan triple-drug blister pack may improve $H$.pylori eradication success through the increase in overall vonoprazan use regardless of separate tablets or combination blister pack.

\section{Proportion of Combination Blister Pack Use for H. pylori Eradication (Secondary Endpoint)}

The use of combination blister packs was defined as the receipt of any combination blister pack containing lansoprazole, rabeprazole, or vonoprazan. Data used for the denominator were those with recorded prescriptions for any first-line clarithromycin triple therapy. This was assessed because the launch of the vonoprazan 
triple-drug blister pack may also have improved $H$. pylori eradication success rates through the increase in combination blister pack use.

\section{Comorbidities}

Comorbidities that may lead to necessitate $H$. pylori eradication were defined on the basis of the World Health Organization International Classification of Diseases, 10th Revision, or Japanese Standard Disease Names (JSDN): peptic ulcer (K25-K28), gastritis (K29.3-K29.5, Helicobacter pylori gastritis [JSDN], intestinal metaplasia [JSDN]), and gastric MALT lymphoma (JSDN) [30, 31]. These were compared between prelaunch and post-launch periods.

\section{Statistical Analysis}

Patient characteristics were expressed as mean (standard deviation [SD]). For ITS, the time series was generated from monthly success rates of clarithromycin triple therapy and proportion of vonoprazan use and proportion of combination blister pack use compared with those who had prescription records for first-line clarithromycin triple therapy. This led to the generation of 25 months of data (observations) in our time series, aligning with the number of months between June 2015 and June 2017. We considered a lag period of 1 month before the true influence of the vonoprazan triple-drug blister pack could be observed on the outcomes because some hospitals did not adopt the combination pack $\left(\right.$ VONOSAP $^{\circledR}$ ) in the launch month; therefore, data in June 2016 were excluded. Monthly success rate and proportion of vonoprazan and of combination blister pack use were then utilized to compare changes between the pre- and post-launch periods of vonoprazan triple-drug blister pack using ITS analysis. The following equation was used for the regression: $Y_{t}=\beta_{0}+\beta_{1} T_{t}+\beta_{2} X_{t}+\beta_{3} X_{t} T_{t}+$ $\varepsilon_{t}$, where $Y_{t}$ is the aggregated proportions measured at month $t ; T_{t}$ is the time since the start of observation (in months); $X_{t}$ is a dummy (indicator) variable representing the launch of vonoprazan triple-drug blister pack (prelaunch periods $=0$, post-launch periods $=1) ;$ and, $X_{t} T_{t}$ is an interaction term. The models were parameterized to test for both a one-time change immediately at the time of implementation (intercept; $\beta_{2}$ ) and the difference in the pre- and post-launch trends (slope; $\beta_{3}$ ). The Durbin-Watson statistic was used to examine for the presence of autocorrelation among serial observations, and the model was corrected for autocorrelation if required [32]. The ITS analysis was conducted in accordance with the Cochrane Effective Practice and Organization of Care Review Group recommendations [33].

We conducted two sensitivity analyses to confirm the robustness of the analysis. First, to determine the effect on inferences instead of a lag period of 1 month, models excluded 3 months between June 2016 and August 2016 to account for possible gradual launch effects on practice. Second, to exclude patients who may have been lost to treatment follow-up, we restricted patients to those who had been tested for $H$. pylori infection at any time after completion of first-line clarithromycin triple therapy of the primary analysis. Procedures or drug codes for the following were considered tests for the confirmation of $H$.pylori eradication: rapid urease test, microbial culture, urea breath test, antibody measurement, and stool antigen test.

A $P$ value less than 0.05 was considered statistically significant. SAS 9.4 (SAS Institute, Cary, USA) and R 3.5.1 (The R Foundation, Vienna, Austria) were used to perform the statistical analyses. The analyses in this study were conducted with no missing data.

\section{RESULTS}

\section{Patient Characteristics}

A total of 36,570 patients received first-line clarithromycin triple therapy during the prelaunch period and 35,721 patients received the same therapy during the post-launch period. Table 1 shows characteristics of the study patients. Mean age of patients was 51.8 (SD 10.3) and 50.9 (SD 10.3) years, respectively; number of women was 15,677 (42.9\%) and $14,748(41.3 \%)$ during prelaunch and postlaunch periods, respectively. 
Table 1 Patient characteristics

\begin{tabular}{lll}
\hline & $\begin{array}{l}\text { Prelaunch } \\
\text { period }^{\mathbf{a}} \\
(\boldsymbol{n}=\mathbf{3 6 , 5 7 0})\end{array}$ & $\begin{array}{l}\text { Post-launch } \\
\text { period }^{\mathbf{b}} \\
(\boldsymbol{n}=\mathbf{3 5}, \mathbf{7 2 1})\end{array}$ \\
\hline Age, years & $51.8 \pm 10.3$ & $50.9 \pm 10.3$ \\
Men/women, $n$ & $20,893 /$ & $20,973 / 14,748$ \\
Peptic ulcer, $n(\%)$ & 15,677 & \\
Gastritis, $n(\%)$ & $31,424(85.9)$ & $31,224(87.4)$ \\
Gastric MALT & $17(0.05)$ & $19(0.05)$ \\
lymphoma, $n(\%)$ & & $11,135(31.2)$ \\
\hline
\end{tabular}

Continuous data are expressed as mean \pm standard deviation

MALT mucosa-associated lymphoid tissue

a Prelaunch period was June 2015 to May 2016

b Post-launch period was July 2016 to June 2017

\section{Success Rate of Clarithromycin Triple Therapy (Primary Endpoint)}

The success rate of first-line clarithromycin triple therapy was $83.1 \%(2574 / 3096)$ in June 2015 , increasing to $92.0 \%(3088 / 3355)$ by June 2017. In June 2016, an immediate change in success rate was observed. The ITS analysis showed a $2.44 \%(95 \%$ CI 1.36-3.52; $P<0.0001)$ increase in clarithromycin triple-therapy success rate from May 2016 to July 2016 (Fig. 2a; Table 2). Slope of regression lines did not show a significant difference between the two periods $(-0.08 \% ; 95 \% \mathrm{CI}-0.22$ to $0.07 ; P=0.30)$. The Durbin-Watson test was not significant at certain lags apart from up to 12 months, suggesting nonsignificant autocorrelation.

\section{Proportion of Vonoprazan Use Relative to Overall PPIs and P-CAB Use for H. pylori Eradication (Secondary Endpoint)}

The proportion of vonoprazan use relative to overall PPI and P-CAB use for H. pylori eradication was $39.9 \%(1235 / 3096)$ in June 2015 , increasing to $84.5 \%(2836 / 3355)$ by June 2017 . In June 2016, an immediate change was observed. The ITS analysis showed a $12.7 \%$
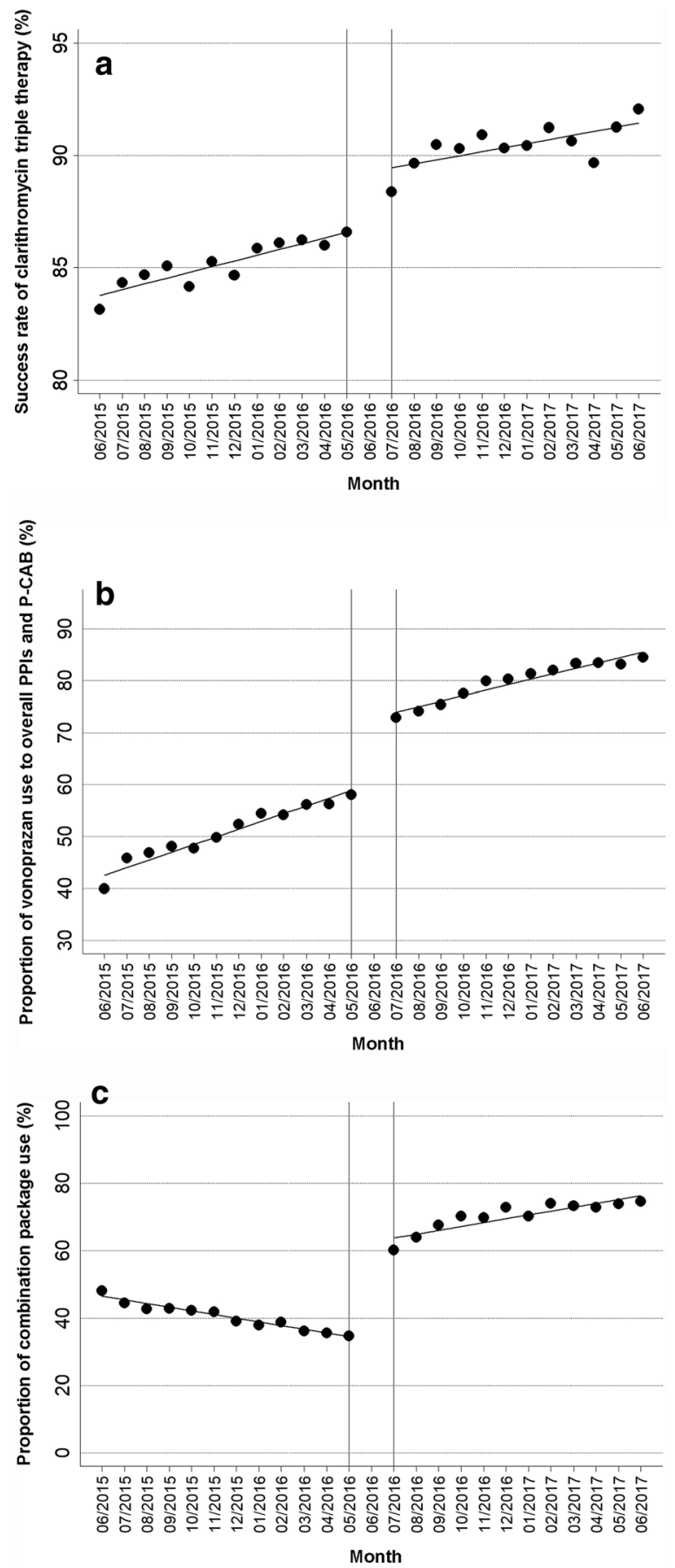

Fig. 2 Outcomes before and after launch of vonoprazan triple-drug blister pack in June 2016. a Success rate of clarithromycin triple therapy. b Proportion of vonoprazan use to overall PPIs and P-CAB use. $\mathbf{c}$ Proportion of tripledrug blister pack use. $P$ - $C A B$ potassium-competitive acid blockers, $P P I$ proton-pump inhibitor 
Table 2 Interrupted time series analysis before and after launch of vonoprazan combination blister pack in June 2016

\begin{tabular}{|c|c|c|c|c|c|c|}
\hline \multirow[t]{2}{*}{ Outcome } & \multirow{2}{*}{$\begin{array}{l}\text { Just before launch } \\
\text { Level at the last } \\
\text { month before } \\
\text { launch, } \%^{\mathrm{a}}\end{array}$} & \multirow{2}{*}{$\begin{array}{l}\text { Just after launch } \\
\text { Level at the first } \\
\text { month after } \\
\text { launch, \% }{ }^{\text {b }}\end{array}$} & \multicolumn{4}{|c|}{$\begin{array}{l}\text { Change associated with launch of vonoprazan } \\
\text { combination package }\end{array}$} \\
\hline & & & $\begin{array}{l}\text { Immediate } \\
\text { change }^{c} \\
(95 \% \mathrm{CI})\end{array}$ & $P$ value & $\begin{array}{l}\text { Change in } \\
\text { slope }(95 \% \\
\text { CI) }\end{array}$ & $P$ value \\
\hline $\begin{array}{l}\text { Success rate of } \\
\text { clarithromycin triple } \\
\text { therapy }\end{array}$ & 86.6 & 88.4 & $\begin{array}{l}2.44(1.36 \text { to } \\
3.52)\end{array}$ & $<0.0001$ & $\begin{array}{r}-0.08 \\
(-0.22 \\
\text { to } 0.07)\end{array}$ & 0.30 \\
\hline $\begin{array}{l}\text { Proportion of vonoprazan } \\
\text { use to overall PPI and } \\
\text { P-CAB use }\end{array}$ & 58.0 & 73.0 & $\begin{array}{l}12.7(10.0 \text { to } \\
15.3)\end{array}$ & $<0.0001$ & $\begin{array}{l}-0.45 \\
(-0.82 \\
\text { to } \\
-0.08)\end{array}$ & 0.03 \\
\hline $\begin{array}{l}\text { Proportion of triple-drug } \\
\text { blister pack use }\end{array}$ & 34.7 & 60.1 & $\begin{array}{l}29.2(25.4 \text { to } \\
32.9)\end{array}$ & $<0.0001$ & $\begin{array}{r}2.24(1.71 \\
\text { to } 2.76)\end{array}$ & $<0.0001$ \\
\hline
\end{tabular}

$C I$ confidence interval, $P$-CAB potassium-competitive acid blockers, $P P I$ proton-pump inhibitor

a The last month before launch was May 2016

b The first month after launch was July 2016

${ }^{c}$ Immediate change represents the intercept, an increase or decrease from May 2016 to July 2016 that is distinct from ongoing trends

(95\% CI 10.0-15.3; $P<0.0001$ ) increase in vonoprazan use proportional to overall PPI and P-CAB use from May 2016 to July 2016 (Fig. 2b; Table 2). Slope of regression lines decreased between the two periods $(-0.45 \%$; $95 \%$ CI -0.82 to $-0.08 ; P=0.03)$. We corrected a firstorder autocorrelation, because the Durbin-Watson test was significant at lag apart from a 1-month period.

\section{Proportion of Combination Blister Pack Use for H. pylori Eradication (Secondary Endpoint)}

The proportion of combination blister pack use for $H$. pylori eradication was $48.2 \%(1491 / 3096)$ in June 2015, increasing to $74.5 \%(2501 / 3355)$ by June 2017. In June 2016, the immediate change was observed. The ITS analysis showed a 29.2\% (95\% CI 25.4-32.9; $P<0.0001$ ) increase in combination blister pack use from May 2016 to July 2016 (Fig. 2c; Table 2). Slope of regression lines also increased between the two periods (2.24\%; 95\% CI 1.71-2.76; $P<0.0001)$. We corrected a first-order autocorrelation, because the Durbin-Watson test was significant at lag apart from a 1-month period.

\section{Sensitivity Analysis}

First, instead of a lag period of 1 month, a model was conducted in which a lag of 3 months from June 2016 to August 2016 was excluded. Inferences were unchanged relative to the main models. The success rate of clarithromycin triple therapy increased by $2.86 \%$ (95\% CI 1.81-3.91; $P<0.0001)$ from May 2016 to September 2016. Slope of regression lines decreased between the two periods $(-0.16 \%$; $95 \%$ CI -0.28 to $-0.03 ; P=0.02$ ). Likewise, the proportion of vonoprazan use increased by $13.6 \%$ (95\% CI 10.6-16.5; $P<0.0001$ ) and slope of regression lines decreased between the two periods $(-0.73 \%$; $95 \% \mathrm{CI}-1.08$ to -0.38 ; $P<0.0001)$. Proportion of blister pack use increased by $37.1 \% \quad(95 \%$ CI $34.9-39.3$; $P<0.0001)$ and slope of regression lines also increased between the two periods (1.77\%; 95\% 
CI 1.50-2.04; $P<0.0001)$. Second, we restricted patients to those who were tested for $H$. pylori infection after receiving first-line clarithromycin triple therapy of the primary analysis. The success rate of clarithromycin triple therapy increased by $3.01 \%$ (95\% CI 1.86-4.17; $P<0.0001$ ) from May 2016 to September 2016. Slope of regression lines did not show a significant difference between the two periods ( $-0.11 \%$; $95 \%$ CI -0.26 to $0.05 ; P=0.17)$.

\section{DISCUSSION}

This is the first study to show an actual change in success rate of clarithromycin triple therapy after vonoprazan triple-drug blister pack launch, by analysis of a large health claims database at an individual level. As hypothesized, the launch of the vonoprazan triple-drug blister pack for $H$. pylori eradication increased the success rate of clarithromycin triple therapy and the proportion of vonoprazan and of combination blister pack use. Moreover, the robust association was confirmed by two sensitivity analyses (one with adjusted lag periods of 3 months, and another in which patients were restricted to those who were tested for $H$. pylori infection after first-line clarithromycin triple therapy). In contrast, the trends (slope) were not markedly different between pre- and postlaunch, and were actually almost flat. This may be because the clarithromycin success rate and the proportion of vonoprazan use reached the practical upper limit.

The results of the current study are consistent with those of previous studies. In the phase 3 clinical trial, the eradication success rate of clarithromycin triple therapy with vonoprazan was $92.6 \%$ and with lansoprazole, a PPI, 75.9\%; noninferiority was confirmed in the primary analysis and superiority was confirmed in an additional analysis [21]. In our study, an immediate increase of $12.7 \%$ was observed in the proportion of vonoprazan use, which may have contributed to the immediate increase in the eradication success rate. Reducing the burden of complex prescribing with clarithromycin triple therapy can mean easier and more accurate administration of medicine, which may be preferable for patients and doctors.

Additionally, the eradication success rate of a lansoprazole triple-drug blister pack group was shown to be higher than that of a separatetablets group in a retrospective study [24]. Although two RCTs demonstrated that the difference in success rate between the groups was not significant, the Hawthorne effect may have impacted the RCTs because both groups had good drug compliance $[25,26]$. In our study, the immediate increase in the proportion of combination blister pack use was $29.2 \%$, which may have led to the immediate increase in the eradication success rate with the increase in the proportion of vonoprazan use. Further studies are needed to evaluate whether drug compliance increases the success rate of $H$. pylori eradication in the real world.

The present study has two limitations. First, because the results of $H$. pylori infection tests were not available in the database, success of clarithromycin triple therapy was defined by lack of second-line therapy after having received first-line clarithromycin triple therapy. This definition may be prone to misclassification bias because those who had failed the first time but decided not to receive second-line therapy were regarded as successfully treated. Nevertheless, the clarithromycin triple therapy success rate of the current study $(87.8 \%)$ was consistent with that of previous studies: 74.8-91.4\% [34-38]. Therefore, we propose that the eradication success rate calculated in this study is comparable to the true eradication success rate. Secondly, we cannot rule out the possibility of bias due to concurrent interventions. For instance, in September 2016, Japanese clinical guidelines for $H$. pylori diagnosis were released, which may have also contributed to increased vonoprazan use. However, this influence is proposed to be limited because the increase was already observed before the guidelines were released.

\section{CONCLUSION}

This is the first study to demonstrate an immediate increase in clarithromycin triple-therapy 
success rate, with a greater proportion of vonoprazan use and a greater proportion of combination blister pack use after the vonoprazan triple-drug blister pack was launched. These findings suggest that introducing easily administered formulations may be effective in changing prescribing practice and subsequent patient outcomes.

\section{ACKNOWLEDGEMENTS}

Funding. Takeda Pharmaceutical Company Limited bought the database from JMDC Inc. Audit of the statistical analysis and English language editing were conducted by TIS (www. tis.jp) and MIMS (www.mimsmc.com), respectively, and were funded by Takeda Pharmaceutical Company Limited. The study, Rapid Service Fee and Open Access Fee were funded by Takeda Pharmaceutical Company Limited.

Medical Writing, Editorial, and Other Assistance. The authors would like to send our appreciation to Kurt Helge Rimkus, Keita Fujikawa, and Ikuo Fujimori from Takeda Pharmaceutical Company Limited for supporting this study. We would also like to thank TIS (www.tis. jp) for audit of the statistical analysis and MIMS (www.mimsmc.com) for English language editing.

Authorship. All named authors meet the International Committee of Medical Journal Editors (ICMJE) criteria for authorship for this article, take responsibility for the integrity of the work as a whole, and have given their approval for this version to be published.

Authorship Contributions. Hisato Deguchi: interpretation of study results; drafting, critical revision, and approval of the final version of the manuscript; involved in the study design and in the statistical analysis. Hajime Yamazaki: interpretation of study results; drafting, critical revision, and approval of the final version of the manuscript; involved in the study design. Tsukasa Kamitani: interpretation of study results; drafting, critical revision, and approval of the final version of the manuscript. Yosuke Yamamoto: interpretation of study results; drafting, critical revision, and approval of the final version of the manuscript. Shunichi Fukuhara: interpretation of study results; drafting, critical revision, and approval of the final version of the manuscript. All authors approved the final version of the article, including the authorship list.

Disclosures. Shunichi Fukuhara received fees for lectures and consulting from Takeda Pharmaceutical Company Limited. Under a contract with Kyoto University, fees for consulting with Hajime Yamazaki were paid Kyoto University, which are not related to this work. Hisato Deguchi is an employee of Takeda Pharmaceutical Company Limited. The other authors declare no conflicts of interest for this article.

Compliance with Ethics Guidelines. The study was conducted according to the Declaration of Helsinki and the International Society for Pharmacoepidemiology Guidelines for Good Pharmacoepidemiology Practices. It was carried out retrospectively using a database of anonymized data, following Ethical Guidelines for Medical and Health Research Involving Human Subjects issued by the Japanese Ministry of Health, Labor, and Welfare [39]. The investigators could only access anonymized information from JMDC database; therefore, in accordance with the Japanese Ethical Guidelines mentioned above, institutional ethics approval and informed consent were not required.

Data Availability. The data that support the findings of this study are available from JMDC Inc. but were used under license for the current study; therefore, restrictions apply, and the data are not publicly available. For inquiries about access to the data set used in this study, please contact JMDC (https://www.jmdc.co.jp).

Open Access. This article is licensed under a Creative Commons Attribution-NonCommercial 4.0 International License, which permits any non-commercial use, sharing, adaptation, distribution and reproduction in any medium or format, as long as you give 
appropriate credit to the original author(s) and the source, provide a link to the Creative Commons licence, and indicate if changes were made. The images or other third party material in this article are included in the article's Creative Commons licence, unless indicated otherwise in a credit line to the material. If material is not included in the article's Creative Commons licence and your intended use is not permitted by statutory regulation or exceeds the permitted use, you will need to obtain permission directly from the copyright holder. To view a copy of this licence, visit http:// creativecommons.org/licenses/by-nc/4.0/.

\section{REFERENCES}

1. Brown LM. Helicobacter pylori: epidemiology and routes of transmission. Epidemiol Rev. 2000;22: 283-97.

2. Everhart JE. Recent developments in the epidemiology of Helicobacter pylori. Gastroenterol Clin North Am. 2000;29:559-78.

3. Ford AC, Axon AT. Epidemiology of Helicobacter pylori infection and public health implications. Helicobacter. 2010;15(Suppl 1):1-6.

4. Malfertheiner P, Megraud F, O'Morain CA, et al. Management of Helicobacter pylori infection-the Maastricht V/florence consensus report. Gut. 2017;66:6-30.

5. Chey WD, Leontiadis GI, Howden CW, Moss SF. ACG clinical guideline: treatment of Helicobacter pylori infection. Am J Gastroenterol. 2017;112: 212-39.

6. Asaka M, Kato M, Takahashi S, et al. Guidelines for the management of Helicobacter pylori infection in Japan: 2009 revised edition. Helicobacter. 2010;15: $1-20$.

7. Fukase K, Kato M, Kikuchi S, et al. Effect of eradication of Helicobacter pylori on incidence of metachronous gastric carcinoma after endoscopic resection of early gastric cancer: an open-label, randomised controlled trial. Lancet. 2008;372: 392-7.

8. Choi IJ, Kook MC, Kim YI, et al. Helicobacter pylori therapy for the prevention of metachronous gastric cancer. N Engl J Med. 2018;378:1085-95.
9. Ford AC, Forman D, Hunt RH, Yuan Y, Moayyedi P. Helicobacter pylori eradication therapy to prevent gastric cancer in healthy asymptomatic infected individuals: systematic review and meta-analysis of randomised controlled trials. BMJ. 2014;348:g3174.

10. Yoon SB, Park JM, Lim CH, Cho YK, Choi MG. Effect of Helicobacter pylori eradication on metachronous gastric cancer after endoscopic resection of gastric tumors: a meta-analysis. Helicobacter. 2014;19:243-8.

11. Chen HN, Wang Z, Li X, Zhou ZG. Helicobacter pylori eradication cannot reduce the risk of gastric cancer in patients with intestinal metaplasia and dysplasia: evidence from a meta-analysis. Gastric Cancer. 2016;19:166-75.

12. Jung DH, Kim JH, Chung HS, et al. Helicobacter pylori eradication on the prevention of metachronous lesions after endoscopic resection of gastric neoplasm: a meta-analysis. PLoS ONE. 2015;10: e0124725.

13. Lee YC, Chiang TH, Chou CK, et al. Association between Helicobacter pylori eradication and gastric cancer incidence: a systematic review and metaanalysis. Gastroenterology. 2016;150:1113-24 (e5).

14. Ford AC, Delaney BC, Forman D, Moayyedi P. Eradication therapy in Helicobacter pylori positive peptic ulcer disease: systematic review and economic analysis. Am J Gastroenterol. 2004;99: 1833-55.

15. Leodolter A, Kulig M, Brasch H, Meyer-Sabellek W, Willich SN, Malfertheiner P. A meta-analysis comparing eradication, healing and relapse rates in patients with Helicobacter pylori-associated gastric or duodenal ulcer. Aliment Pharmacol Ther. 2001;15: 1949-58.

16. Sugano K. Effect of Helicobacter pylori eradication on the incidence of gastric cancer: a systematic review and meta-analysis. Gastric Cancer. 2019;22: 435-45.

17. Thung I, Aramin H, Vavinskaya V, et al. Review article: the global emergence of Helicobacter pylori antibiotic resistance. Aliment Pharmacol Ther. 2016;43:514-33.

18. Kawai T, Takahashi S, Suzuki H, et al. Changes in the first line Helicobacter pylori eradication rates using the triple therapy-a multicenter study in the Tokyo metropolitan area (Tokyo Helicobacter pylori study group). J Gastroenterol Hepatol. 2014;29(Suppl 4):29-32.

19. Deguchi H, Uda A, Murakami K. Current status of Helicobacter pylori diagnosis and eradication therapy 
in japan using a nationwide database. Digestion. 2019. https://doi.org/10.1159/000500819.

20. Mori H, Suzuki H, Omata F, et al. Current status of first- and second-line Helicobacter pylori eradication therapy in the metropolitan area: a multicenter study with a large number of patients. Therap Adv Gastroenterol. 2019;12:1756284819858511.

21. Murakami K, Sakurai Y, Shiino M, Funao N, Nishimura A, Asaka M. Vonoprazan, a novel potassiumcompetitive acid blocker, as a component of firstline and second-line triple therapy for Helicobacter pylori eradication: a phase III, randomised, doubleblind study. Gut. 2016;65:1439-46.

22. Takeda Pharmaceutical Company Ltd. 2016. https://www.takeda.com/newsroom/newsreleases/ 2016/vonosap-pack-400-vonosap-pack-800-andvonopion-pack-triple-drug-blister-packscontaining-takecab-tablet-now-available-forhelicobacter-pylori-eradication-in-japan/. Accessed May 11, 2020.

23. Sasaki M, Ogasawara N, Utsumi K, et al. The effectiveness of packed therapy with three drugs in Helicobacter pylori eradication in Japan. Methods Find Exp Clin Pharmacol. 2010;32:243-6.

24. Nishida T, Tsujii Y, Okamoto A, et al. A triple-drug blister-packaged drug with vonoprazan improves first-line eradication of Helicobacter pylori in elderly patients: a retrospective propensity score-matched cohort study. Digestion. 2020;101:608-14.

25. Kawai T, Kawakami K, Kataoka M, et al. The effectiveness of packaged medicine in eradication therapy of Helicobacter pylori in Japan. J Clin Biochem Nutr. 2006;38:73-6.

26. Nagahara A, Miwa H, Hojo M, et al. Efficacy of Lansap combination therapy for eradication of H. pylori. Helicobacter. 2007;12:643-4.

27. Harris AD, McGregor JC, Perencevich EN, et al. The use and interpretation of quasi-experimental studies in medical informatics. J Am Med Inform Assoc. 2006;13:16-23.

28. Bernal JL, Cummins S, Gasparrini A. Interrupted time series regression for the evaluation of public health interventions: a tutorial. Int J Epidemiol. 2017;46:348-55.

29. Deguchi H, Yamazaki H, Yamamoto Y, Fukuhara S. Association between parental history of Helicobacter pylori treatment failure and treatment failure in the offspring. J Gastroenterol Hepatol. 2019. https:// doi.org/10.1111/jgh.14774.

30. Quan H, Sundararajan V, Halfon P, et al. Coding algorithms for defining comorbidities in ICD-9-CM and ICD-10 administrative data. Med Care. 2005;43:1130-9.

31. Japanese Standard Disease Names. http://www. byomei.org/information/index.html. Accessed Mar $11,2020$.

32. Durbin J, Watson GS. Testing for serial correlation in least squares regression. II Biometrika. 1951;38: 159-78.

33. Data collection checklist-Cochrane Effective Practice and Organisation of Care Review Group (EPOC). http://epoc.cochrane.org/sites/epoc. cochrane.org/files/public/uploads/ datacollectionchecklist.pdf. Accessed May 11, 2020.

34. Li BZ, Threapleton DE, Wang JY, et al. Comparative effectiveness and tolerance of treatments for Helicobacter pylori: systematic review and network metaanalysis. BMJ. 2015;351:h4052.

35. Jung YS, Kim EH, Park CH. Systematic review with meta-analysis: the efficacy of vonoprazan-based triple therapy on Helicobacter pylori eradication. Aliment Pharmacol Ther. 2017;46:106-14.

36. Dong SQ, Singh TP, Wei X, Yao H, Wang HL. Review: a Japanese population-based meta-analysis of vonoprazan versus PPI for Helicobacter pylori eradication therapy: Is superiority an illusion? Helicobacter. 2017;22:e12438.

37. Li M, Oshima T, Horikawa T, et al. Systematic review with meta-analysis: Vonoprazan, a potent acid blocker, is superior to proton-pump inhibitors for eradication of clarithromycin-resistant strains of Helicobacter pylori. Helicobacter. 2018;23:e12495.

38. Lyu QJ, Pu QH, Zhong XF, Zhang J. Efficacy and safety of vonoprazan-based versus proton pump inhibitor-based triple therapy for Helicobacter pylori eradication: a meta-analysis of randomized clinical trials. Biomed Res Int. 2019;2019:9781212.

39. Ministry of Health Law. Ethical guidelines for medical and health research involving human subjects 2015. https://www.mhlw.go.jp/file/06Seisakujouhou-10600000Daijinkanboukouseikagakuka/0000080278.pdf. Accessed Mar 2021. 